\title{
RELATIVISTIC ROTATIONAL EFFECTS: APPLICATION TO THE EARTH-MOON SYSTEM
}

\author{
DAVID VOKROUHLICKÝ \\ Observatoire de la Côte d'Azur, Dept. CERGA, \\ Av N. Copernic, 06130 Grasse, France ${ }^{1}$
}

\begin{abstract}
Relativistic spin effects involved in the Earth-Moon dynamics are reviewed. They enclose: (i) the coordinate system effects, and (ii) the relativistic physical librations. The geodetic precession is the only relativistic spin phenomenon which has been firmly detected so far. The best candidates of the effects which might be detected in the forthcoming period are the lunar physical librations and coordinate nutations. As for the latter, however, a fine cancellation between the geodetic and the LenseThirring coordinate effects results in decreasing their amplitude just below the possibility of the Lunar Laser Ranging technology.
\end{abstract}

\section{Introduction}

The relativity theory became a well-established tool for the Solar system studies. Planetary and satellite dynamics, reference frame as well as time scales definition need to account for tiny but observable relativistic effects. Small values of "the compactness parameter" (relating the gravitational radius and the true radius of a given body) and "the slow motion" (expressed in units of the light velocity) indicate that using a first post-Newtonian approximation (1PN) is appropriate in the Solar system. Exception is reserved for a group of proposed high-precision experiments which need to account for the higher order effects. These will not be discussed in this text.

The most exhaustive and ambitious approach to the 1PN dynamics of the system of $N$ extended bodies has been recently presented by Damour

\footnotetext{
${ }^{1}$ On leave from the Institute of Astronomy, Charles University Prague, Švédská 8,
} 15000 Prague 5, The Czech Republic. 
et al. $(1991,1992,1993,1994)$ [hereafter called DSX]. Not only the theory accounts in an elegant way for all details of the multipole structure of the gravity field(s) but also harbors in a natural way the theory of the local and global coordinate systems and time scales. We base our approach on the DSX series of papers.

In what follows, we discuss the relativistic spin effects in the Earth-Moon system with special care for the lunar motion. Thanks to a high precision of the Lunar Laser Ranging (LLR) technology we dispose of a data of superior quality if compared to the orbital motion data of the other solar system bodies $^{2}$. This places the lunar motion among a good candidates for testing the relativistic effects.

Indeed, the lunar motion gives now the best quantitative evidence for the weak equivalence principle [see, for instance, discussion in Damour and Vokrouhlický (1995)]. Also, a high quality constraints on the 1PN parameters $\beta$ and $\gamma$, as well as variation of the gravitational constant $\dot{G} / G$ are available [e.g. Williams et al. (1995)]. Finally, a special interest for our discussion is due to about $1 \%$ verification of the de Sitter (geodetic) precession through the secular rate of the lunar longitude of perigee [Williams et al. (1995)]. Formally, it can be understood as "the relativistic spin" effect related to the local Earth-Moon reference frame definition, although the interpretation considering this phenomenon as a relativistic perturbation of the lunar orbital motion is equally possible [this is the way how historically de Sitter (1916) discovered the effect].

In the next section, we give an outlook for possibilities to measure another relativistic spin effects in the Earth-Moon system in the forthcoming period.

\section{1PN rotational dynamics and its application to the Earth- Moon system}

In the third paper of the DSX series the authors succeeded to derive 1PN equations of the rotational motion relating the local-time $T_{A}$ derivative of the properly defined spin vector $S_{a}^{A}$ of body A to the perturbing torques

$$
\frac{d S_{a}^{A}}{d T_{A}}=\sum_{l \geq 0} \frac{1}{l !} \epsilon_{a b c}\left[M_{b L}^{A} G_{c L}^{A}+\frac{1}{c^{2}} \frac{l+1}{l+2} S_{b L}^{A} H_{c L}^{A}\right]+\mathcal{O}\left(c^{-4}\right) .
$$

The first term in the bracket describes coupling of the body A massmultipoles $M_{L}^{A}$ with the gravitoelectric multipoles $G_{L}^{A}$ of the external grav-

\footnotetext{
${ }^{2}$ The best current LLR performance is 1 to 2 centimeters as a formal error of "the normal points" for the lunar motion. Technological improvements are supposed to suppress this precision to about 3 millimeters in the forthcoming years (C. Veillet, private communication).
} 
itational field measured in the local frame of body A. The latter split into a piece which can be considered as "formally Newtonian" and a 1PN correction. The lowest order 1PN effect related to these terms comes from putting $l=1$ in Eq. (1) and results from coupling of the mass quadrupole-moment $M_{a b}^{A}\left[\right.$ dominated by the flattening parameter $\left.J_{2}^{A}\right]$ with the $1 \mathrm{PN}$ piece of the gravitoelectric field $G_{a b}^{A}$. Corresponding relativistic "librations" has been recently examined for the Earth [Bizouard et al. (1992)] and for the Moon [Bois and Vokrouhlický (1995)]. In the former case their amplitude is very small, but in the lunar case it reaches the order of 1 milliarcsecond. The latter value might be in the range of detection possibilities of the improved LLR technology in the next years.

The presence of the $1 / c^{2}$ factor in front of the second term in the right hand side of Eq. (1) clearly points out that it is entirely the 1PN order. Of these terms, the most important corresponds to fixing $l=0$. It can be formally interpreted as the local coordinate system precession with the angular velocity given by the appropriate piece of the gravitomagnetic field $H_{a}\left(T_{A}\right)$. The latter quantity depends on the external gravitational potentials (both the scalar one, resulting in the geodetic precession, and the vectorial one, resulting in the Lense-Thirring precession) measured at the origin of the local frame of a given body $\mathrm{A}$ and also on the acceleration of the body A (resulting in the Thomas precession). Such secular relativistic rotational effects called "precession" are also accompanied by the periodic ones called "nutations". In the following, we briefly review recent calculations of these phenomena for the Earth and Moon referentials [see for details Fukushima (1991), Brumberg et al. (1992), Vokrouhlický (1995)].

The effects arising from the interaction of the solar gravity field with the Earth-Moon center-of-mass motion are the same for both the Earth and Moon referentials and, thus, can be attributed to the Earth-Moon centerof-mass referential system. They enclose the (observed) geodetic precession with the rate of 1919.4 mas/cy. Of particular interest is the Lense-Thirring (LT) precession, because this important prediction of the general relativity theory has not been experimentally verified yet. Careful analysis shows, that there are two sorts of LT precession: (i) the first related to the solar gravity field coupling with the Earth-Moon total angular momentum having the rate of about $0.47 \mathrm{mas} / \mathrm{cy}$, and (ii) the second given by the solar angular momentum having the rate of about $-0.28 \mathrm{mas} / \mathrm{cy}$. It is interesting to note that some recent works, pioneered by Brumberg et al. (1992), prefer to join the former LT effect to the definition of "the geodetic precession constant" (in the broader sense). The reason is that this piece of the LT precession can be calculated with sufficient precision contrary to the latter effect which is submitted to at most $50 \%$ error due to not precisely known value of the total angular momentum of the Sun. More detailed discussion 
can be found in Vokrouhlický (1995). Apart from the common terms, the mutual Earth-Moon dynamics results in the individual precession of the lunar and terrestrial reference frames. About $29.8 \mathrm{mas} / \mathrm{cy}$ advance of the lunar frame represents a major effect of these terms. The effect is potentially "observable" using the forthcoming year LLR data, but its secular nature troubles it decorrelation from the effects of the tidal origin. On contrary, it might be suggested to subtract this value from the lunar rotation in longitude to achieve a better precision of the tidal parameters adjustment.

The lunar librations associated with these coordinate effects has been studied by Vokrouhlický (1995). As a result of a fine cancellation of the geodetic and LT phenomena their magnitude is unfortunately small (approximately 0.03 mas). Individually, both effects are one order of magnitude greater (but opposite sign), about the same as the principal annual term discovered by Fukushima (1991). A wide spectrum of these relativistic nutations has been computed by Brumberg et al. (1992) for the terrestrial referential. Generally their amplitudes are of the order of microarcseconds (or smaller). Necessity of their use will attend the next generation of the astrometric projects.

Acknowledgement. The author worked on this paper when staying at OCA/CERGA (France) thanks to the H. Poincaré research grant.

\section{References}

Bizouard, C., Schastok, J., Soffel, M. and Souchay, J. (1992) Etude de la rotation de la Terre dans la cadre de la relativite generale: Premier approche, in: Journee 1992: Systemes de reference spatio-temporels ed. N. Capitaine, Paris Observatory, pp. 7684.

Bois, E. and Vokrouhlický, D. (1995) The relativistic spin effects in the Earth-Moon system, Astron. Astrophys., 300, p. 559.

Brumberg, V.A., Bretagnon, P. and Francou, G. (1991) Analytical algorithms of relativistic reduction of astronomical observations, in: Journee 1991: Systemes de reference spatio-temporels ed. N. Capitaine, Paris Observatory, pp. 141-148.

Damour, T. (1987) chapter 6 of 300 years of gravitation, eds. S.W. Hawking and W. Israel, Cambridge Univ. Press, Cambridge, pp. 128-198.

Damour, T. and Vokrouhlický, D. (1995), The equivalence principle and the Moon, Phys. Rev. $D$, in press.

Damour, T., Soffel, M. and Xu, C. (1991) Phys. Rev. D, 43, p. 3273; (1992) Phys. Rev. D, 45, p. 1017; (1993) Phys. Rev. D, 47, p. 1017; (1994) Phys. Rev. D, 49, p. 618; see also Damour, T. and Vokrouhlický, D. (1995) Phys. Rev. D, 52, p. 4455.

Dickey, J.O. et al., Science, 265, p. 482.

Fukushima, T. (1991) Astron. Astrophys., 244, p. L11.

Sitter, W. de (1916) Mon. Not. R. astr. Soc., 76, p. 699; (1916) Mon. Not. R. astr. Soc., 77, p. 155.

Vokrouhlický, D. (1995) The relativistic spin effects in the Earth-Moon system, Phys. Rev. $D$, in press.

Williams, J.G., Newhall, X.X. and Dickey, J.O. (1995) Relativity parameters determined from Lunar Laser Ranging, Phys. Rev. D, in press. 